\title{
ON SCHRÖDINGER EQUATIONS WITH INDEFINITE NONLINEARITIES
}

\author{
JING LONG and JIANFU YANG ${ }^{凶}$
}

(Received 29 May 2008)

\begin{abstract}
In this paper we consider the problem of finding standing waves - solutions to nonlinear Schrödinger equations with vanishing potential and sign-changing nonlinearities. This involves searching for solutions of the problem

$$
-\varepsilon^{2} \Delta u+V(x) u=Q(x)|u|^{p-1} u \quad \text { in } \mathbb{R}^{N} .
$$

We show that the problem has a solution, and the maximum point of the solution is concentrated on a minimum point of some function as $\varepsilon \rightarrow 0$.
\end{abstract}

2000 Mathematics subject classification: primary 35J20, 35B40.

Keywords and phrases: Schrödinger equation, weighted Sobolev space, concentration.

\section{Introduction}

In this paper we are concerned with the nonlinear Schrödinger equation

$$
i h \frac{\partial \psi}{\partial t}=-\frac{h^{2}}{2 m} \Delta_{x} \psi+V(x) \psi-Q(x)|\psi|^{p-1} \psi,
$$

where $m$ and $h$ are positive constants, $\psi: \mathbb{R}^{+} \times \mathbb{R}^{N} \rightarrow \mathbb{C}, V \in C\left(\mathbb{R}^{N}, \mathbb{R}\right)$. One of the basic principles of quantum mechanics states that it contains classical mechanics as its limit as $h \rightarrow 0$. The presence of the nonlinear term in the equation makes possible the appearance of solitary waves. It is also interesting in mathematics to analyze the behavior of solutions of (1.1) as $h \rightarrow 0$. There has been much attention in the literature to the so-called standing waves, namely solutions of (1.1) of the form $\psi(x, t)=e^{-(i E t) / h} u(x)$, where $E$ is some real constant and $u(x)$ is real-valued. This is the simplest form of solitary wave. After conveniently relabeling the parameters, $u(x)$ satisfies

$$
-\varepsilon^{2} \Delta u+V(x) u=Q(x)|u|^{p-1} u \quad \text { in } \mathbb{R}^{N},
$$

This work was supported by National Natural Sciences Foundations of China, No: 10571175 and 10631030.

(C) 2009 Australian Mathematical Society 0004-9727/2009 \$16.00 
where $\varepsilon>0$ is a parameter. We assume in this paper that $N \geq 3,1<p<$ $(N+2) /(N-2)$. The potential $V$ vanishes at infinity, and the coefficient $Q$ is signchanging, that is $Q=Q^{+}-Q^{-}, Q^{ \pm} \not \equiv 0$.

In the case where $V$ and $Q$ are positive, the problem has been studied in $[2,7,9,10]$ and references therein. In particular, it is shown in $[9,10]$ that positive ground state solutions of (1.2) are concentrated at a global minimum point of the function

$$
\mathcal{A}(x):=V^{(p+1) /(p-1)-N / 2}(x) Q^{-2 /(p-1)}(x) \quad \text { as } \varepsilon \rightarrow 0 .
$$

A important ingredient of the proof is the monotonicity of critical values with respect to the parameters related to the coefficients $V$ and $Q$. Similar results are obtained in [2] for the case where both $V(x)$ and $Q(x)$ vanish at infinity.

On the other hand, if $Q$ is sign-changing, various existence results are obtained in, for example, [1,4]. The main difficulties are that, firstly, the negative part $Q^{-}$of $Q$ will push the level of the associated energy functional up, making it hard to verify that the functional has minimax geometry if the variational method is applied; secondly, the boundedness of the Palai-Smale sequence is difficult to show due to the presence of $Q^{-}$. This is why a 'thickness' condition or a nondegeneracy condition on the set where $Q=0$ has been required in previous work. Various techniques are then developed to deal with the problem.

In this paper we assume that both $V$ and $Q$ vanish at infinity, and $Q$ is also signchanging. To be precise, we suppose that:

$\left(\mathrm{H}_{1}\right) V \in C\left(\mathbb{R}^{N}\right)$, and there exist $a>0, A>0, \alpha>0$ such that

$$
\frac{a}{1+|x|^{\alpha}} \leq V(x) \leq A
$$

$\left(\mathrm{H}_{2}\right) Q \in C\left(\mathbb{R}^{N}\right)$ is sign-changing, the set $\Omega^{+}:=\left\{x \in \mathbb{R}^{N} \mid Q(x)>0\right\}$ is bounded, $\lim _{|x| \rightarrow \infty} Q(x)<0$, and there exist $C>0$ and $\beta>0$ such that

$$
0<Q^{-}(x) \leq \frac{C}{1+|x|^{\beta}} ;
$$

$\left(\mathrm{H}_{3}\right) \sigma \leq p<(N+2) /(N-2)$, where

$$
\sigma= \begin{cases}\frac{N+2}{N-2}-\frac{4 \beta}{\alpha(N-2)} & \text { if } 0<\beta<\alpha \\ 1 & \text { otherwise. }\end{cases}
$$

We shall show that there exist nonnegative solutions of (1.2), which belong to $H^{1}\left(\mathbb{R}^{N}\right)$ and are concentrated at a global minimum point of

$$
\mathcal{B}(x):=V^{(p+1) /(p-1)-N / 2}(x)\left(Q^{+}\right)^{-2 /(p-1)}(x) .
$$

Since the potential decays to zero at infinity, the variational theory in $H^{1}\left(\mathbb{R}^{N}\right)$ cannot be employed. Inspired by [2], we shall work in the weighted Sobolev space 
$H_{\varepsilon}$, defined by

$$
H_{\varepsilon}=\left\{u \in \mathscr{D}^{1,2}\left(\mathbb{R}^{N}\right) \mid \int_{\mathbb{R}^{N}}\left(\varepsilon^{2}|\nabla u|^{2}+V(x) u^{2}\right) d x<\infty\right\} .
$$

$H_{\varepsilon}$ is a Hilbert space with norm induced by the inner product

$$
(u, v)_{\varepsilon}=\int_{\mathbb{R}^{N}}\left(\varepsilon^{2} \nabla u \cdot \nabla v+V(x) u v\right) d x .
$$

We also denote by $L_{Q^{-}}^{p+1}\left(\mathbb{R}^{N}\right)$ the weighted $L^{p}$ space with norm

$$
\|u\|_{p+1, Q^{-}}^{p+1}=\int_{\mathbb{R}^{N}} Q^{-}(x)|u|^{p+1} d x .
$$

We begin with an existence result.

THEOREM 1.1. Suppose that $\left(\mathrm{H}_{1}\right)-\left(\mathrm{H}_{3}\right)$ hold with $\alpha \in(0,2), \beta>0$. Then for every $\varepsilon>0$ problem (1.2) has a nonnegative classical solution $u_{\varepsilon} \in H^{1}\left(\mathbb{R}^{N}\right)$.

Next, we investigate the limiting behavior of solutions $u_{\varepsilon}$ obtained in Theorem 1.1 as $\varepsilon \rightarrow 0$. We remark that critical values of the associated functional described in Theorem 1.1 are not monotone in parameters related to the coefficients $V$ and $Q$ as critical values in [10], hence the arguments in $[9,10]$ cannot applied. Nevertheless, the following result holds.

THEOREM 1.2. Suppose that $\left(\mathrm{H}_{1}\right)-\left(\mathrm{H}_{3}\right)$ hold with $\alpha \in(0,2), \beta>0$. Then the solution $u_{\varepsilon}$ has a unique maximum $x_{\varepsilon} \in \Omega^{+}$when $\varepsilon>0$ is sufficiently small. Moreover, $u_{\varepsilon}$ is concentrated at a global minimum $x^{*}$ of $\mathcal{A}(x)$, that is, $x_{\varepsilon} \rightarrow x^{*}$ as $\varepsilon \rightarrow 0$ and

$$
u_{\varepsilon}(x)=U^{*}(x)\left(\frac{x-x_{\varepsilon}}{\varepsilon}\right)+w_{\varepsilon}(x)
$$

where $w_{\varepsilon} \rightarrow 0$ in $C_{\mathrm{loc}}^{2}\left(\mathbb{R}^{N}\right)$ as $\varepsilon \rightarrow 0$, and $U^{*}$ is the unique positive solution of

$$
-\Delta U^{*}(x)+V\left(x^{*}\right) U^{*}(x)=Q\left(x^{*}\right)\left(U^{*}\right)^{p}(x) \text { in } \mathbb{R}^{N} .
$$

In Section 2, the applying mountain-pass theorem, we find a nonnegative solution of (1.2) in $H_{\varepsilon}$, and then we show that it belongs to $H^{1}\left(\mathbb{R}^{N}\right)$. In Section 3 we discuss the concentration phenomenon of $u_{\varepsilon}$, and prove Theorem 1.2.

\section{Existence results}

In this section, we establish the existence results for problem (1.2), that is, we prove Theorem 1.1. Solutions of problem (1.2) will be found as critical points of the associated functional

$$
I_{\varepsilon}(u)=\frac{1}{2} \int_{\mathbb{R}^{N}}\left(\varepsilon^{2}|\nabla u|^{2}+V(x) u^{2}\right) d x-\frac{1}{p+1} \int_{\mathbb{R}^{N}} Q(x) u_{+}^{p+1} d x,
$$

in $H_{\varepsilon}$. By the following result, which was discussed in [2], we see that $I_{\varepsilon}$ is well defined and $C^{1}$ in $H_{\varepsilon}$. 
Lemma 2.1. Suppose that $\left(\mathrm{H}_{1}\right)$ and $\left(\mathrm{H}_{2}\right)$ hold with $\alpha \in(0,2]$ and $\beta>0$. Then for all $\varepsilon>0$, the inclusion $H_{\varepsilon} \hookrightarrow L_{Q^{-}}^{p+1}$ is continuous provided that $\sigma \leq p$ $\leq(N+2) /(N-2)$; and it is compact provided that $\sigma<p<(N+2) /(N-2)$.

To look for critical points of $I_{\varepsilon}$, we use the mountain-pass lemma in [8]. It is apparent that $I_{\varepsilon}$ has a mountain-pass geometry in $H_{\varepsilon}$, that is, there exist $\rho, \sigma>0$, such that $I_{\varepsilon}(u) \geq \sigma$ for $\|u\|=\rho$, and there exists $e \in H_{\varepsilon}$ such that for $t \geq 1, I_{\varepsilon}(t e)<0$. By the mountain-pass theorem without the $(P S)_{c}$ condition, there is a $(P S)_{c_{\varepsilon}}$ sequence $\left\{u_{n}\right\}$ of $I_{\varepsilon}$, that is,

$$
I_{\varepsilon}\left(u_{n}\right) \rightarrow c_{\varepsilon}, \quad I_{\varepsilon}^{\prime}\left(u_{n}\right) \rightarrow 0
$$

where

$$
c_{\varepsilon}:=\inf _{\gamma \in \Gamma_{\varepsilon}} \max _{0 \leq t \leq 1} I_{\varepsilon}(\gamma(t)) \geq \sigma
$$

and $\Gamma_{\varepsilon}=\left\{\gamma \in C\left([0,1], H_{\varepsilon}\right) \mid \gamma(0)=0, \gamma(1)=e\right\}$.

PROPOSITION 2.1. Assume that $\left(\mathrm{H}_{1}\right)-\left(\mathrm{H}_{3}\right)$ hold with $0<\alpha \leq 2, \beta>0$. Then for all $\varepsilon>0$, problem (1.2) has at least a nonnegative solution in $H_{\varepsilon}$.

PROOF. It is sufficient to show that the $(P S)_{c_{\varepsilon}}$ sequence $\left\{u_{n}\right\}$ possesses a convergent subsequence. Obviously, for any fixed $\varepsilon>0,\left\{u_{n}\right\}$ is uniformly bounded in $H_{\varepsilon}$. So we may assume that $u_{n} \rightarrow u_{\varepsilon}$ in $H_{\varepsilon}, u_{n} \rightarrow u_{\varepsilon}$ in $L_{\text {loc }}^{q}\left(\mathbb{R}^{N}\right)$ for $2 \leq q<2 N /(N-2)$ and $u_{n} \rightarrow u_{\varepsilon}$ almost everywhere in $\mathbb{R}^{N}$. By the Brezis-Lieb lemma [3],

$$
\begin{aligned}
o(1)+\left\langle I_{\varepsilon}^{\prime}\left(u_{n}\right), u_{n}\right\rangle= & \int_{\mathbb{R}^{N}}\left(\varepsilon^{2}\left|\nabla u_{n}\right|^{2}+V(x) u_{n}^{2}\right) d x-\int_{\mathbb{R}^{N}} Q(x)\left(u_{n}\right)_{+}^{p+1} d x \\
= & \left\langle I_{\varepsilon}^{\prime}\left(u_{\varepsilon}\right), u_{\varepsilon}\right\rangle+\left\|u_{n}-u_{\varepsilon}\right\|_{\varepsilon}^{2}-\int_{\Omega^{+}} Q^{+}(x)\left(u_{n}-u_{\varepsilon}\right)_{+}^{p+1} d x \\
& +\int_{\mathbb{R}^{N}} Q^{-}(x)\left(u_{n}-u_{\varepsilon}\right)_{+}^{p+1} d x .
\end{aligned}
$$

It follows from $\left\langle I_{\varepsilon}^{\prime}\left(u_{\varepsilon}\right), u_{\varepsilon}\right\rangle=0$ that

$$
\left\|u_{n}-u_{\varepsilon}\right\|_{\varepsilon}^{2}+\int_{\mathbb{R}^{N}} Q^{-}(x)\left(u_{n}-u_{\varepsilon}\right)_{+}^{p+1} d x=\int_{\Omega^{+}} Q^{+}(x)\left(u_{n}-u_{\varepsilon}\right)_{+}^{p+1} d x+o(1) \text {. }
$$

As $\Omega^{+}$is bounded, $\int_{\Omega^{+}} Q^{+}(x)\left(u_{n}-u_{\varepsilon}\right)_{+}^{p+1} d x \rightarrow 0$ as $n \rightarrow \infty$. This implies that $u_{n} \rightarrow u_{\varepsilon}$ in $H_{\varepsilon}$.

In the rest of this section, following the idea of [2], we show that $u_{\varepsilon}$ obtained in Proposition 2.1 belongs to $H^{1}\left(\mathbb{R}^{N}\right)$. Since $\Omega^{+}$is bounded, there exists $R_{0}>0$ such that $\Omega^{+} \subset B_{R_{0}}$. In the sequel, we always assume that $0<\alpha<2$.

LEMMA 2.2. For $R>R_{0}$ and $\Omega_{n, \varepsilon} \subset \mathbb{R}^{N} \backslash B_{R}$,

$$
\int_{\Omega_{n+1, \varepsilon}}\left(\varepsilon^{2}\left|\nabla u_{\varepsilon}\right|^{2}+V(x) u_{\varepsilon}^{2}\right) d x \leq \frac{1}{2} \int_{\Omega_{n, \varepsilon}}\left(\varepsilon^{2}\left|\nabla u_{\varepsilon}\right|^{2}+V(x) u_{\varepsilon}^{2}\right) d x,
$$

where $\Omega_{n, \varepsilon}=\mathbb{R}^{N} \backslash B_{R_{n, \varepsilon}}, R_{n, \varepsilon}=\varepsilon n^{2 /(2-\alpha)}$. 
PROOF. Let $\chi_{n, \varepsilon}(r)$ be a cut-off function, $\chi_{n, \varepsilon}(r)=0$ for $r \leq R_{n, \varepsilon}, \chi_{n, \varepsilon}(r)=1$ for $r \geq R_{n+1, \varepsilon}$, and $\left|\nabla \chi_{n, \varepsilon}(x)\right| \leq C /\left(R_{n+1, \varepsilon}-R_{n, \varepsilon}\right)$. By the definition of $R_{n, \varepsilon}$,

$$
\begin{aligned}
\left|R_{n+1, \varepsilon}-R_{n, \varepsilon}\right| & =\varepsilon\left[(n+1)^{2 /(2-\alpha)}-n^{2 /(2-\alpha)}\right] \geq C \varepsilon(n+1)^{\alpha /(2-\alpha)} \\
& =C \varepsilon^{(2-\alpha) / 2} R_{n+1, \varepsilon}^{\alpha / 2} \geq C \varepsilon R_{n+1, \varepsilon}^{\alpha / 2} .
\end{aligned}
$$

This implies that

$$
\varepsilon^{2}\left|R_{n+1, \varepsilon}-R_{n, \varepsilon}\right|^{-2} \leq C R_{n+1, \varepsilon}^{-\alpha} \leq C \inf \left\{V(x): R_{n, \varepsilon} \leq|x| \leq R_{n+1, \varepsilon}\right\},
$$

and

$$
\varepsilon^{2}\left|\nabla \chi_{n, \varepsilon}(x)\right|^{2} \leq V(x)
$$

Expanding $\left\langle I_{\varepsilon}^{\prime}\left(u_{\varepsilon}\right), \chi_{n, \varepsilon} u_{\varepsilon}\right\rangle=0$ and noting that $\Omega^{+} \subset B_{R_{0}}$, for all $R>R_{0}$,

$$
\begin{aligned}
& \int_{\Omega_{n, \varepsilon}} \chi_{n, \varepsilon}\left(\varepsilon^{2}\left|\nabla u_{\varepsilon}\right|^{2}+V(x) u_{\varepsilon}^{2}\right) d x \\
& \quad=\int_{\Omega_{n, \varepsilon}} \chi_{n, \varepsilon} Q(x)\left|u_{\varepsilon}\right|^{p+1} d x-\varepsilon^{2} \int_{\Omega_{n, \varepsilon}} \nabla u_{\varepsilon} \cdot \nabla \chi_{n, \varepsilon} u_{\varepsilon} \\
& \quad \leq \frac{\varepsilon^{2}}{2} \int_{\Omega_{n, \varepsilon}}\left(\left|\nabla u_{\varepsilon}\right|^{2}+\left|\nabla \chi_{n, \varepsilon}\right|^{2} u_{\varepsilon}^{2}\right) d x \\
& \quad \leq \frac{1}{2} \int_{\Omega_{n, \varepsilon}}\left(\varepsilon^{2}\left|\nabla u_{\varepsilon}\right|^{2}+V(x) u_{\varepsilon}^{2}\right) d x,
\end{aligned}
$$

which yields the result.

Lemma 2.3. For all $\rho>4 R_{0}$,

$$
\begin{aligned}
& \int_{\{|x|>\rho\}}\left(\varepsilon^{2}\left|\nabla u_{\varepsilon}\right|^{2}+V(x) u_{\varepsilon}^{2}\right) d x \\
& \quad \leq C\left\|u_{\varepsilon}\right\|_{\varepsilon}^{2} \exp \left\{-\frac{1}{2}\left|\log \frac{1}{2}\right| \varepsilon^{-1}\left(\rho^{(2-\alpha) / 2}-R_{0}^{(2-\alpha) / 2}\right)\right\},
\end{aligned}
$$

where $C>0$ is a constant.

Proof. Given $\rho>4 R_{0}$, let $\tilde{n}-\bar{n}$ be positive integers such that

$$
R_{\bar{n}, \varepsilon} \leq R_{0} \leq R_{\bar{n}+1, \varepsilon}, \quad R_{\widetilde{n}-1, \varepsilon} \leq \rho \leq R_{\widetilde{n}, \varepsilon} .
$$

Choosing $R_{0}$ large enough, and hence $\bar{n}$ large, $R_{\bar{n}+1, \varepsilon}<2 R_{\bar{n}, \varepsilon}$. Therefore, $\rho>4 R_{0} \geq$ $4 R_{\bar{n}, \varepsilon}>R_{\bar{n}+2, \varepsilon}, \widetilde{n}-1 \geq \bar{n}+2$ and $\tilde{n}-\bar{n}-2 \geq 1$. From Lemma 2.2, we deduce that 


$$
\begin{aligned}
& \int_{\{|x|>\rho\}}\left(\varepsilon^{2}\left|\nabla u_{\varepsilon}\right|^{2}+V(x) u_{\varepsilon}^{2}\right) d x \\
& \quad \leq \int_{\left\{|x|>R_{\tilde{n}-1, \varepsilon\}}\right.}\left(\varepsilon^{2}\left|\nabla u_{\varepsilon}\right|^{2}+V(x) u_{\varepsilon}^{2}\right) d x \\
& \leq\left(\frac{1}{2}\right)^{\tilde{n}-\bar{n}-2} \int_{\left\{|x|>R_{\bar{n}+1, \varepsilon}\right\}}\left(\varepsilon^{2}\left|\nabla u_{\varepsilon}\right|^{2}+V(x) u_{\varepsilon}^{2}\right) d x \\
& \leq\left(\frac{1}{2}\right)^{\tilde{n}-\bar{n}-2} \int_{\left\{|x|>R_{0}\right\}}\left(\varepsilon^{2}\left|\nabla u_{\varepsilon}\right|^{2}+V(x) u_{\varepsilon}^{2}\right) d x \\
& \leq\left(\frac{1}{2}\right)^{\tilde{n}-\bar{n}-2}\left\|u_{\varepsilon}\right\|_{\varepsilon}^{2} .
\end{aligned}
$$

By $(2.1), \widetilde{n}-\bar{n} \geq(1 / 2) \varepsilon^{-1}\left(\rho^{(2-\alpha) / 2}-R_{0}^{(2-\alpha) / 2}\right)$, and the assertion follows.

Proof OF THEOREM 1.1. The proof will be complete once we show that $u_{\varepsilon} \in H^{1}\left(\mathbb{R}^{N}\right)$.

Let $y \in \mathbb{R}^{N}$ be such that $|y|>2$. Then

$$
\int_{B_{1}(y)} u_{\varepsilon}^{2} d x=\int_{B_{1}(y)} V(x) u_{\varepsilon}^{2} \frac{1}{V(x)} d x \leq C_{4}|y|^{\alpha} \int_{B_{1}(y)} V(x) u_{\varepsilon}^{2} d x .
$$

For $R=|y| / 2$, since $B_{1}(y) \subset \mathbb{R}^{N} \backslash B_{R}$,

$$
\int_{B_{1}(y)} V(x) u_{\varepsilon}^{2} d x \leq \int_{\mathbb{R}^{N} \backslash B_{R}(0)} V(x) u_{\varepsilon}^{2} d x .
$$

By Lemma 2.3, for $|y|>4 R_{0}$, we obtain

$$
\int_{B_{1}(y)} u_{\varepsilon}^{2} d x \leq C_{4}|y|^{\alpha} \int_{\mathbb{R}^{N} \backslash B_{R}} V(x) u_{\varepsilon}^{2} d x \leq C_{5}\left\|u_{\varepsilon}\right\|_{\varepsilon}^{2}|y|^{\alpha} \exp \left\{-C_{6}|y|^{1-(\alpha / 2)}\right\} .
$$

Let $\left\{y_{i}\right\} \subset B_{5} \backslash B_{2}, i=1,2, \ldots, m, m \in \mathbb{N}$, be such that $B_{5} \backslash B_{2} \subset \bigcup_{i=1}^{m} B_{1}\left(y_{i}\right)$. Let $y_{i, k}:=2^{k} y_{i}$. We may assume that there exists $k_{0}$ such that $2^{k_{0}}>4 R_{0}$. By Lemma 2.3 and noticing that $\mathbb{R}^{N} \backslash B_{2} \subset \bigcup_{k=0}^{\infty} 2^{k}\left(B_{5} \backslash B_{2}\right)$,

$$
\begin{aligned}
\int_{\mathbb{R}^{N} \backslash B_{2}} u_{\varepsilon}^{2} d x \leq & \sum_{k=0}^{\infty} \int_{2^{k}\left(B_{5} \backslash B_{2}\right)} u_{\varepsilon}^{2} d x \leq \sum_{i=1}^{m} \sum_{k=0}^{\infty} \int_{B_{2^{k}}\left(y_{i, k}\right)} u_{\varepsilon}^{2} d x \\
= & \sum_{i=1}^{m} \sum_{k=0}^{k_{0}-1} \int_{B_{2^{k}}\left(y_{i, k}\right)} u_{\varepsilon}^{2} d x+\sum_{i=1}^{m} \sum_{k=k_{0}}^{\infty} \int_{B_{2^{k}}\left(y_{i, k}\right)} u_{\varepsilon}^{2} d x \\
\leq & \sum_{i=1}^{m} \sum_{k=0}^{k_{0}-1} \int_{B_{2^{k}}\left(y_{i, k}\right)} u_{\varepsilon}^{2} d x+C_{5}\left\|u_{\varepsilon}\right\|_{\varepsilon}^{2} \sum_{i=1}^{m} \sum_{k=k_{0}}^{\infty}\left|y_{i, k}\right|^{\alpha} \\
& \times \exp \left\{-C_{6}\left|y_{i, k}\right|^{1-(\alpha / 2)}\right\},
\end{aligned}
$$


which yields $\int_{\mathbb{R}^{N} \backslash B_{2}} u_{\varepsilon}^{2} d x<+\infty$, since $0<\alpha<2$. Thus $u_{\varepsilon} \in L^{2}\left(\mathbb{R}^{N}\right)$ and hence $u_{\varepsilon} \in H^{1}\left(\mathbb{R}^{N}\right)$. Finally, standard arguments show that $u_{\varepsilon} \in C^{2}\left(\mathbb{R}^{N}\right)$ and $u_{\varepsilon} \geq 0$.

\section{Concentration phenomenon}

In this section, we discuss the limit behavior of the solution $u_{\varepsilon}$ of (1.2) obtained in Theorem 1.1.

LEMMA 3.1. Let $x_{\varepsilon}$ be a global maximum of $u_{\varepsilon}$, that is, $u_{\varepsilon}\left(x_{\varepsilon}\right)=\max _{x \in \mathbb{R}^{N}} u_{\varepsilon}(x)$. Then $x_{\varepsilon} \in \Omega^{+}$.

PROOF. Since $x_{\varepsilon}$ is a global maximum of $u_{\varepsilon}$, then $\Delta u_{\varepsilon}\left(x_{\varepsilon}\right) \leq 0$. The maximum principle implies that $u_{\varepsilon}\left(x_{\varepsilon}\right)>0$ and $-\Delta u_{\varepsilon}\left(x_{\varepsilon}\right)+V\left(x_{\varepsilon}\right) u_{\varepsilon}\left(x_{\varepsilon}\right)>0$. Supposing, on the other hand, that $x_{\varepsilon} \in \mathbb{R}^{N} \backslash \Omega^{+}$, we obtain

$$
-\Delta u_{\varepsilon}\left(x_{\varepsilon}\right)+V\left(x_{\varepsilon}\right) u_{\varepsilon}\left(x_{\varepsilon}\right)=Q\left(x_{\varepsilon}\right) u_{\varepsilon}^{p+1}\left(x_{\varepsilon}\right) \leq 0,
$$

a contradiction.

We define for $\xi \in \Omega^{+}$the functional $F_{\xi}$ on $H^{1}\left(\mathbb{R}^{N}\right)$ by

$$
F_{\xi}(u)=\frac{1}{2} \int_{\mathbb{R}^{N}}\left(|\nabla u|^{2}+V(\xi) u^{2}\right) d x-\frac{1}{p+1} Q(\xi) \int_{\mathbb{R}^{N}}|u|^{p+1} d x .
$$

Let

$$
f(\xi)=\inf _{\mathcal{N}_{\xi}} F_{\xi}(u)
$$

where

$$
\mathcal{N}_{\xi}=\left\{\left.u \in H^{1}\left(\mathbb{R}^{N}\right) \backslash\{0\}\left|\int_{\mathbb{R}^{N}}\left(|\nabla u|^{2}+V(\xi) u^{2}\right) d x=Q(\xi) \int_{\mathbb{R}^{N}}\right| u\right|^{p+1} d x\right\} .
$$

Then $u \in \mathcal{N}_{\xi}$ if and only if $\tilde{u}(y):=Q^{1 /(p-1)}(\xi) V^{-1 /(p-1)}(\xi) u\left(V^{-1 / 2}(\xi) y\right) \in \mathcal{N}$, where

$$
\mathcal{N}=\left\{\left.u \in H^{1}\left(\mathbb{R}^{N}\right) \backslash\{0\}\left|\int_{\mathbb{R}^{N}}\left(|\nabla u|^{2}+u^{2}\right) d x=\int_{\mathbb{R}^{N}}\right| u\right|^{p+1} d x\right\} .
$$

Therefore,

$$
\begin{aligned}
f(\xi) & =\inf _{\mathcal{N}_{\xi}} F_{\xi}(u)=\left(\frac{1}{2}-\frac{1}{p+1}\right) Q(\xi) \inf _{u \in \mathcal{N}_{\xi}} \int_{\mathbb{R}^{N}}|u|^{p+1} d x \\
& =\left(\frac{1}{2}-\frac{1}{p+1}\right) Q^{-2 /(p-1)}(\xi) V^{(p+1) /(p-1)-N / 2}(\xi) \inf _{v \in \mathcal{N}} \int_{\mathbb{R}^{N}}|v|^{p+1} d x
\end{aligned}
$$

Recall that $\mathcal{B}(x)=V^{(p+1) /(p-1)-N / 2}(x)\left(Q^{+}\right)^{-2 /(p-1)}(x)$. 
LEMma 3.2. There exists $C_{0}>0$ such that for all $\xi \in \Omega^{+}$and all $\varepsilon$ sufficiently small,

$$
\varepsilon^{-N} c_{\varepsilon}=\varepsilon^{-N} I_{\varepsilon}\left(u_{\varepsilon}\right) \leq C_{0} \mathcal{B}(\xi)+o(1)
$$

as $\varepsilon \rightarrow 0$. In particular, there exists $C>0$ such that $c_{\varepsilon} \leq C \varepsilon^{N},\left\|u_{\varepsilon}\right\|_{\varepsilon}^{2} \leq C \varepsilon^{N}$.

Proof. Let $U \in H^{1}\left(\mathbb{R}^{N}\right)$ be the unique positive radial solution of

$$
-\Delta U+U=U^{p} \quad \text { in } \mathbb{R}^{N} .
$$

Since $\inf _{v \in \mathcal{N}} \int_{\mathbb{R}^{N}}|v|^{p+1} d x$ is achieved by $U$, we obtain

$$
f(\xi)=\left(\frac{1}{2}-\frac{1}{p+1}\right) Q^{-2 /(p-1)}(\xi) V^{(p+1) /(p-1)-N / 2}(\xi) \int_{\mathbb{R}^{N}}|U|^{p+1} d x=C_{0} \mathcal{A}(\xi) .
$$

It is known that $f(\xi)$ is a mountain-pass level of $F_{\xi}$, so for all $v>0$ there exists $w \in H^{1}\left(\mathbb{R}^{N}\right)$ such that

$$
f(\xi) \leq \max _{t>0} F_{\xi}(t w) \leq f(\xi)+v .
$$

Let $\phi \in C^{2}\left(\mathbb{R}^{N}\right)$ be a cut-off function such that $0 \leq \phi \leq 1$ and $\phi \equiv 1$ in a neighborhood of $\xi$, and define $w_{\varepsilon} \in H^{1}\left(\mathbb{R}^{N}\right)$ by $w_{\varepsilon}(x)=\phi(x) w(x-\xi / \varepsilon)$. It follows from $H^{1}\left(\mathbb{R}^{N}\right) \subset H_{\varepsilon}$ that $w_{\varepsilon} \in H_{\varepsilon}$, and

$$
\begin{aligned}
\varepsilon^{-N}\left\|w_{\varepsilon}\right\|_{\varepsilon}^{2}= & \varepsilon^{2} \int_{\mathbb{R}^{N}}|\nabla \phi(\varepsilon y+\xi)|^{2} w^{2}(y) d y+2 \varepsilon \\
& \times \int_{\mathbb{R}^{N}} \nabla w(y) \nabla \phi(\varepsilon y+\xi) w(y) \phi(\varepsilon y+\xi) d y \\
& +\int_{\mathbb{R}^{N}} \phi^{2}(\varepsilon y+\xi)|\nabla w(y)|^{2} d y \\
& +\int_{\mathbb{R}^{N}} V(\varepsilon y+\xi) \phi^{2}(\varepsilon y+\xi) w^{2}(y) d y
\end{aligned}
$$

and

$$
\varepsilon^{-N} \int_{\mathbb{R}^{N}} Q(x)\left|w_{\varepsilon}\right|^{p+1} d x=\int_{\mathbb{R}^{N}} Q(\varepsilon y+\xi)|\phi(\varepsilon y+\xi) w(y)|^{p+1} d y .
$$

Thus we obtain

$$
\varepsilon^{-N} I_{\varepsilon}\left(t w_{\varepsilon}\right)=F_{\xi}(t w)+o(1)
$$

as $\varepsilon \rightarrow 0$. As a result,

$$
\begin{aligned}
\varepsilon^{-N} I_{\varepsilon}\left(u_{\varepsilon}\right) & \leq \inf _{v \in H_{\varepsilon} \backslash\{0\}} \max _{t>0} \varepsilon^{-N} I_{\varepsilon}(t v) \leq \max _{t>0} \varepsilon^{-N} I_{\varepsilon}\left(t w_{\varepsilon}\right) \\
& \leq \max _{t>0} F_{\xi}(t w)+o(1) \leq f(\xi)+v+o(1) \\
& =C_{0} \mathcal{B}(\xi)+v+o(1) .
\end{aligned}
$$


Since $v>0$ is arbitrary,

$$
\varepsilon^{-N} c_{\varepsilon}=\varepsilon^{-N} I_{\varepsilon}\left(u_{\varepsilon}\right) \leq C_{0} \mathcal{B}(\xi)+o(1) .
$$

In particular, $c_{\varepsilon} \leq \varepsilon^{N} C_{0} \inf _{\xi \in \Omega^{+}} \mathcal{B}(\xi) \leq C \varepsilon^{N}$ and $\left\|u_{\varepsilon}\right\|_{\varepsilon}^{2} \leq C \varepsilon^{N}$.

LemMa 3.3. There exists a constant $C>0$ such that $\left\|u_{\varepsilon}\right\|_{L^{\infty}} \geq C$ for all $\varepsilon>0$ sufficiently small.

PROOF. Apparently,

$$
\left\|u_{\varepsilon}\right\|_{\varepsilon}^{2}=\int_{\mathbb{R}^{N}}\left(\varepsilon^{2}\left|\nabla u_{\varepsilon}\right|^{2}+V(x) u_{\varepsilon}^{2}\right) d x=\int_{\mathbb{R}^{N}} Q(x) u_{\varepsilon}^{p+1} d x .
$$

Moreover,

$$
\int_{\mathbb{R}^{N}} Q(x) u_{\varepsilon}^{p+1} d x \leq \int_{\Omega^{+}} Q^{+}(x) u_{\varepsilon}^{p+1} d x \leq \int_{B_{R_{0}}} Q^{+}(x) u_{\varepsilon}^{p+1} d x .
$$

By $\left(\mathrm{H}_{1}\right),\left(\mathrm{H}_{2}\right)$,

$$
Q^{+} \leq C \leq \frac{C}{a}\left(1+|x|^{\alpha}\right) V(x) \leq \frac{C}{a}\left(1+R_{0}^{\alpha}\right) V(x),
$$

for $|x| \leq R_{0}$. Therefore,

$$
\int_{B_{R_{0}}} Q^{+}(x) u_{\varepsilon}^{2} d x \leq \frac{C}{a}\left(1+R_{0}^{\alpha}\right) \int_{B_{R_{0}}} V(x) u_{\varepsilon}^{2} d x \leq \frac{C}{a}\left(1+R_{0}^{\alpha}\right)\left\|u_{\varepsilon}\right\|_{\varepsilon}^{2} .
$$

By (3.3), (3.4) and (3.5), we obtain

$$
\begin{aligned}
\left\|u_{\varepsilon}\right\|_{\varepsilon}^{2} & =\int_{\mathbb{R}^{N}} Q(x) u_{\varepsilon}^{p+1} d x \leq \int_{B_{R_{0}}} Q^{+}(x) u_{\varepsilon}^{p+1} d x \\
& \leq\left\|u_{\varepsilon}\right\|_{L^{\infty}}^{p-1} \int_{B_{R_{0}}} Q^{+}(x) u_{\varepsilon}^{2} d x \\
& \leq \frac{C}{a}\left(1+R_{0}^{\alpha}\right)\left\|u_{\varepsilon}\right\|_{\varepsilon}^{2}\left\|u_{\varepsilon}\right\|_{L^{\infty}}^{p-1} .
\end{aligned}
$$

This implies that $\left\|u_{\varepsilon}\right\|_{L^{\infty}}^{p-1} \geq a /\left(C\left(1+R_{0}^{\alpha}\right)\right)>0$ and $\left\|u_{\varepsilon}\right\|_{L^{\infty}} \geq C$.

Proof of Theorem 1.2. We know from Lemma 3.1 that $x_{\varepsilon} \in \Omega^{+}$, so we may assume that $x_{\varepsilon} \rightarrow x^{*} \in \overline{\Omega^{+}}$as $\varepsilon \rightarrow 0$. Let $v_{\varepsilon}(x):=u_{\varepsilon}\left(\varepsilon x+x_{\varepsilon}\right)$. Then $v_{\varepsilon}$ satisfies

$$
-\Delta v_{\varepsilon}(x)+V\left(\varepsilon x+x_{\varepsilon}\right) v_{\varepsilon}(x)=Q\left(\varepsilon x+x_{\varepsilon}\right) v_{\varepsilon}^{p}, \quad x \in \mathbb{R}^{N} .
$$


By Lemma 3.2 and assumption $\left(\mathrm{H}_{1}\right)$,

$$
\begin{aligned}
C & \geq \varepsilon^{-N}\left\|u_{\varepsilon}\right\|_{\varepsilon}^{2}=\varepsilon^{-N} \int_{\mathbb{R}^{N}}\left(\varepsilon^{2}\left|\nabla u_{\varepsilon}\right|^{2}+V(x) u_{\varepsilon}^{2}\right) d x \\
& \geq \varepsilon^{-N} \int_{\mathbb{R}^{N}}\left(\varepsilon^{2}\left|\nabla u_{\varepsilon}\right|^{2}+\frac{a}{1+|x|^{\alpha}} u_{\varepsilon}^{2}\right) d x \\
& =\int_{\mathbb{R}^{N}}\left(\left|\nabla v_{\varepsilon}(y)\right|^{2}+\frac{a}{1+\left|\varepsilon y+x_{\varepsilon}\right|^{\alpha}} v_{\varepsilon}^{2}(y)\right) d y \\
& \geq C \int_{\mathbb{R}^{N}}\left(\left|\nabla v_{\varepsilon}(y)\right|^{2}+\frac{a}{1+|y|^{\alpha}} v_{\varepsilon}^{2}(y)\right) d y
\end{aligned}
$$

since $\left|\varepsilon y+x_{\varepsilon}\right| \leq C(1+|y|)$. Therefore,

$$
\int_{\mathbb{R}^{N}}\left(\left|\nabla v_{\varepsilon}(y)\right|^{2}+\frac{a}{1+|y|^{\alpha}} v_{\varepsilon}^{2}(y)\right) d y \leq C,
$$

where $C>0$ is independent of $\varepsilon$. By this fact, we may verify that $\left\{v_{\varepsilon}\right\}_{\varepsilon}$ is uniformly bounded in $H_{\mathrm{loc}}^{1}\left(\mathbb{R}^{N}\right)$ and $C_{\mathrm{loc}}^{2, \alpha}\left(\mathbb{R}^{N}\right)$ with respect to $\varepsilon$, and we may assume that, up to a subsequence, $v_{\varepsilon} \rightarrow U^{*}$ in $C_{\text {loc }}^{2, \alpha}\left(\mathbb{R}^{N}\right)$. Passing to the limit in Equation (3.6), we find that $U^{*} \geq 0$ is a classical solution of the problem

$$
-\Delta U^{*}(x)+V\left(x^{*}\right) U^{*}(x)=Q\left(x^{*}\right)\left(U^{*}\right)^{p}(x) \quad \text { in } \mathbb{R}^{N} .
$$

By Lemma 3.3, $\max _{x \in \mathbb{R}^{N}} v_{\varepsilon}(x)=v_{\varepsilon}(0)=u_{\varepsilon}\left(x_{\varepsilon}\right)=\left\|u_{\varepsilon}\right\|_{L^{\infty}} \geq C>0$. It follows that $\max _{x \in \mathbb{R}^{N}} U^{*}(x)=U^{*}(0) \geq C>0$. If $x^{*} \in \partial \Omega^{+}$, then $Q\left(x^{*}\right)=0,-\Delta U^{*}(x)$ $+V\left(x^{*}\right) U^{*}(x)=0$, by the maximum principle we have $U^{*}(x) \equiv 0$, it is impossible. Thus, $x^{*} \in \Omega^{+}$. For any sequence $R_{n} \rightarrow+\infty$,

$$
\int_{\bar{B}_{R_{n}}}\left(\left|\nabla v_{\varepsilon}\right|^{2}+V\left(\varepsilon x+x_{\varepsilon}\right) v_{\varepsilon}^{2}\right) d x \leq \varepsilon^{-N}\left\|u_{\varepsilon}\right\|_{\varepsilon}^{2} \leq C .
$$

Since $v_{\varepsilon} \rightarrow U^{*}$ in $C^{2, \alpha}\left(\bar{B}_{R_{n}}\right)$ for fixed $n$, the dominated convergence theorem implies that

$$
\int_{\bar{B}_{R_{n}}}\left(\left|\nabla U^{*}\right|^{2}+V\left(x^{*}\right)\left(U^{*}\right)^{2}\right) d x \leq C .
$$

Letting $R_{n} \rightarrow+\infty$, we deduce that $U^{*} \in H^{1}\left(\mathbb{R}^{N}\right)$. By the maximum principle, $U^{*}>0$ in $\mathbb{R}^{N}$, and a result in [5] implies that $U^{*}$ is a radial function.

We claim that

$$
F_{x^{*}}\left(U^{*}\right) \leq \liminf _{\varepsilon \rightarrow 0} \varepsilon^{-N} I_{\varepsilon}\left(u_{\varepsilon}\right)
$$

Indeed, if

$$
h_{\varepsilon}(x):=\frac{1}{2}\left|\nabla v_{\varepsilon}\right|^{2}+\frac{1}{2} V\left(\varepsilon x+x_{\varepsilon}\right) v_{\varepsilon}^{2}(x)-\frac{1}{p+1} Q\left(\varepsilon x+x_{\varepsilon}\right) v_{\varepsilon}^{p+1},
$$


then $I_{\varepsilon}\left(u_{\varepsilon}\right)=\varepsilon^{N} \int_{\mathbb{R}^{N}} h_{\varepsilon}(x) d x$. Since $v_{\varepsilon} \rightarrow U^{*}$ in $C_{\text {loc }}^{2, \alpha}\left(\mathbb{R}^{N}\right)$, for all $R>0$,

$$
\begin{aligned}
\lim _{\varepsilon \rightarrow 0} \int_{B_{R}} h_{\varepsilon}(x) d x= & \frac{1}{2} \int_{B_{R}}\left|\nabla U^{*}\right|^{2} d x+\frac{1}{2} V\left(x^{*}\right) \int_{B_{R}}\left(U^{*}\right)^{2} d x \\
& -\frac{1}{p+1} Q\left(x^{*}\right) \int_{B_{R}}\left(U^{*}\right)^{p+1} d x .
\end{aligned}
$$

Since $U^{*} \in H^{1}\left(\mathbb{R}^{N}\right)$, for any $v>0$, we can choose $R>0$ large enough such that

$$
\begin{aligned}
\lim _{\varepsilon \rightarrow 0} \int_{B_{R}} h_{\varepsilon}(x) d x & \geq \int_{\mathbb{R}^{N}}\left[\frac{1}{2}\left|\nabla U^{*}\right|^{2}+\frac{1}{2} V\left(x^{*}\right)\left(U^{*}\right)^{2}-\frac{1}{p+1} Q\left(x^{*}\right)\left(U^{*}\right)^{p+1}\right] d x-v \\
& =F_{x^{*}}\left(U^{*}\right)-v .
\end{aligned}
$$

Let $\eta_{R}$ be a cut-off function such that $\eta_{R}=0$ in $B_{R-1}, \quad \eta_{R}=1$ in $\mathbb{R}^{N} \backslash B_{R}$, $0 \leq \eta_{R} \leq 1,\left|\nabla \eta_{R}\right| \leq C$. Testing (3.6) with $\eta_{R} v_{\varepsilon}$, we obtain

$$
\int_{\mathbb{R}^{N} \backslash B_{R}}\left(\left|\nabla v_{\varepsilon}\right|^{2}+V(x) v_{\varepsilon}^{2}\right) d x-\int_{\mathbb{R}^{N} \backslash B_{R}} Q\left(\varepsilon x+x_{\varepsilon}\right) v_{\varepsilon}^{p+1} d x+E_{\varepsilon}=0,
$$

where

$$
E_{\varepsilon}:=\int_{B_{R} \backslash B_{R-1}}\left[\nabla v_{\varepsilon} \cdot \nabla\left(\eta_{R} v_{\varepsilon}\right)+V\left(\varepsilon x+x_{\varepsilon}\right) \eta_{R} v_{\varepsilon}^{2}-Q\left(\varepsilon x+x_{\varepsilon}\right) \eta_{R} v_{\varepsilon}^{p+1}\right] d x
$$

Hence,

$$
0 \leq \int_{\mathbb{R}^{N} \backslash B_{R}}\left(\left|\nabla v_{\varepsilon}\right|^{2}+V(x) v_{\varepsilon}^{2}\right) d x=\int_{\mathbb{R}^{N} \backslash B_{R}} Q\left(\varepsilon x+x_{\varepsilon}\right) v_{\varepsilon}^{p+1} d x-E_{\varepsilon}
$$

and

$$
\int_{\mathbb{R}^{N} \backslash B_{R}} Q\left(\varepsilon x+x_{\varepsilon}\right) v_{\varepsilon}^{p+1} d x \geq E_{\varepsilon}
$$

Thus

$$
\begin{aligned}
\int_{\mathbb{R}^{N} \backslash B_{R}} h_{\varepsilon}(x) d x= & \frac{1}{2} \int_{\mathbb{R}^{N} \backslash B_{R}}\left(\left|\nabla v_{\varepsilon}\right|^{2}+V\left(\varepsilon x+x_{\varepsilon}\right) v_{\varepsilon}^{2}(x)\right) d x \\
& -\frac{1}{p+1} \int_{\mathbb{R}^{N} \backslash B_{R}} Q\left(\varepsilon x+x_{\varepsilon}\right) v_{\varepsilon}^{p+1} d x \\
= & \left(\frac{1}{2}-\frac{1}{p+1}\right) \int_{\mathbb{R}^{N} \backslash B_{R}} Q\left(\varepsilon x+x_{\varepsilon}\right) v_{\varepsilon}^{p+1} d x-\frac{1}{2} E_{\varepsilon} \\
\geq & -\frac{1}{p+1} E_{\varepsilon}
\end{aligned}
$$


For $R$ large enough, $\lim _{\varepsilon \rightarrow 0}\left|E_{\varepsilon}\right| \leq \nu$. Indeed, since $v_{\varepsilon} \rightarrow U^{*}$ in $C_{\text {loc }}^{2, \alpha}\left(\mathbb{R}^{N}\right)$,

$$
\begin{gathered}
\lim _{\varepsilon \rightarrow 0} \int_{B_{R} \backslash B_{R-1}}\left(\nabla v_{\varepsilon} \cdot \nabla\left(\eta_{R} v_{\varepsilon}\right)+V\left(\varepsilon x+x_{\varepsilon}\right) \eta_{R} v_{\varepsilon}^{2}\right) d x \\
\leq C \int_{B_{R} \backslash B_{R-1}}\left(\left|\nabla U^{*}\right|^{2}+\nabla U^{*} U^{*}+V\left(x^{*}\right)\left(U^{*}\right)^{2}\right) d x \\
\lim _{\varepsilon \rightarrow 0} \int_{B_{R} \backslash B_{R-1}} Q\left(\varepsilon x+x_{\varepsilon}\right) \eta_{R} v_{\varepsilon}^{p+1} d x \leq \int_{B_{R} \backslash B_{R-1}} Q\left(x^{*}\right)\left(U^{*}\right)^{p+1} d x .
\end{gathered}
$$

For $R$ large enough, $U^{*} \in H^{1}\left(\mathbb{R}^{N}\right)$ implies that

$$
\begin{gathered}
C \int_{B_{R} \backslash B_{R-1}}\left(\left|\nabla U^{*}\right|^{2}+\nabla U^{*} U^{*}+V\left(x^{*}\right)\left(U^{*}\right)^{2}\right) d x \leq v / 2, \\
\int_{B_{R} \backslash B_{R-1}} Q\left(x^{*}\right)\left(U^{*}\right)^{p+1} d x \leq v / 2 .
\end{gathered}
$$

Thus $\lim _{\varepsilon \rightarrow 0}\left|E_{\varepsilon}\right| \leq v$. This yields

$$
\liminf _{\varepsilon \rightarrow 0} \int_{\mathbb{R}^{N} \backslash B_{R}} h_{\varepsilon}(x) d x \geq-\frac{v}{2} .
$$

From (3.9) and (3.10) we obtain

$$
\liminf _{\varepsilon \rightarrow 0} \int_{\mathbb{R}^{N}} h_{\varepsilon}(x) d x \geq F_{x^{*}}\left(U^{*}\right)-\frac{3}{2} \nu
$$

for any $v>0$. Inequality (3.8) then follows.

Now we show that $x^{*}$ is a global minimum of the function $f(\xi)=C_{0} \mathcal{A}(\xi)$. Suppose that it is not a global minimum. There would exist $\xi^{*} \in \Omega^{+}$such that $f\left(x^{*}\right)>f\left(\xi^{*}\right)$. By (3.8) and Lemma 3.2, for all $\xi \in \Omega^{+}$,

$$
F_{x^{*}}\left(U^{*}\right) \leq \liminf _{\varepsilon \rightarrow 0} \varepsilon^{-N} I_{\varepsilon}\left(u_{\varepsilon}\right) \leq C_{0} \mathcal{A}(\xi) .
$$

Since $U^{*}$ is a solution of (3.7), we infer that

$$
F_{x^{*}}\left(U^{*}\right) \geq \inf _{\mathcal{N}_{x^{*}}} F_{x^{*}}(u)=f\left(x^{*}\right)>f\left(\xi^{*}\right)=C_{0} \mathcal{A}\left(\xi^{*}\right),
$$

which yields a contradiction.

It remains to show that $u_{\varepsilon}$ has at most one maximum point if $\varepsilon$ is sufficiently small. Suppose, by way of contradiction, that $u_{\varepsilon}$ has another maximum point $z_{\varepsilon} \in \Omega^{+}$which is different from $x_{\varepsilon}$. We may assume that $z_{\varepsilon} \rightarrow z^{*} \in \Omega^{+}$. Noticing that $\varepsilon^{-1}\left(z_{\varepsilon}-x_{\varepsilon}\right)$ is also a maximum point of $v_{\varepsilon}$, there are two cases which may occur:

(i) $\varepsilon^{-1}\left(z_{\varepsilon}-x_{\varepsilon}\right)$ is bounded;

(ii) $\varepsilon^{-1}\left(z_{\varepsilon}-x_{\varepsilon}\right)$ is unbounded. 
In case (i), we assume, up to a subsequence, that $\varepsilon^{-1}\left(z_{\varepsilon}-x_{\varepsilon}\right) \rightarrow P \in \mathbb{R}^{N}$ as $\varepsilon \rightarrow 0$. Since $v_{\varepsilon}\left(\varepsilon^{-1}\left(z_{\varepsilon}-x_{\varepsilon}\right)\right)=\max v_{\varepsilon}$ converges to max $U^{*}=U^{*}(0)$, we conclude that $P=0$. Therefore, for $\varepsilon$ sufficiently small, $\varepsilon^{-1}\left(z_{\varepsilon}-x_{\varepsilon}\right) \in B_{r}$, which is impossible since by [6, pp. 836-837], 0 is the only critical point of $v_{\varepsilon}$ in $B_{r}$.

In case (ii), let $\widehat{v}_{\varepsilon}(x):=u_{\varepsilon}\left(\varepsilon x+z_{\varepsilon}\right)$. To prove $v_{\varepsilon} \rightarrow U^{*}$ in $C_{\text {loc }}^{2, \alpha}\left(\mathbb{R}^{N}\right)$, we may show that $\widehat{v}_{\varepsilon} \rightarrow \widehat{U}^{*}$ in $C_{\text {loc }}^{2, \alpha}\left(\mathbb{R}^{N}\right)$, and $\widehat{U}^{*} \in H^{1}\left(\mathbb{R}^{N}\right)$ is the unique positive radial solution of

$$
-\Delta \widehat{U}^{*}(x)+V\left(z^{*}\right) \widehat{U}^{*}(x)=Q\left(z^{*}\right)\left(\widehat{U}^{*}\right)^{p}(x) \text { in } \mathbb{R}^{N}
$$

Since $\left|\varepsilon^{-1}\left(z_{\varepsilon}-x_{\varepsilon}\right)\right| \rightarrow \infty$, then for $\varepsilon$ sufficiently small and for any $R>0$ fixed, $\bar{B}_{R} \cap \bar{B}^{\varepsilon}=\emptyset$, where $\bar{B}^{\varepsilon}:=\bar{B}_{R}\left(\varepsilon^{-1}\left(z_{\varepsilon}-x_{\varepsilon}\right)\right)$. We may deduce as in the proof of (3.9) and (3.10) that for any $v>0$, there exists $R>0$ large enough such that

$$
\lim _{\varepsilon \rightarrow 0} \int_{\bar{B}^{\varepsilon}} h_{\varepsilon}(x) d x \geq F_{z^{*}}\left(\widehat{U}^{*}\right)-v
$$

and

$$
\liminf _{\varepsilon \rightarrow 0} \int_{\mathbb{R}^{N} \backslash\left(B_{R} \cup B^{\varepsilon}\right)} h_{\varepsilon}(x) d x \geq-v .
$$

It follows from (3.9), (3.11) and (3.12) that

$$
\liminf _{\varepsilon \rightarrow 0} \int_{\mathbb{R}^{N}} h_{\varepsilon}(x) d x \geq F_{x^{*}}\left(U^{*}\right)+F_{z^{*}}\left(\widehat{U}^{*}\right)-3 v .
$$

Lemma 3.2 yields that

$$
F_{x^{*}}\left(U^{*}\right)+F_{z^{*}}\left(\widehat{U}^{*}\right) \leq \liminf _{\varepsilon \rightarrow 0} \int_{\mathbb{R}^{N}} h_{\varepsilon}(x) d x=\liminf _{\varepsilon \rightarrow 0} \varepsilon^{-N} I_{\varepsilon}\left(u_{\varepsilon}\right) \leq f\left(x^{*}\right) .
$$

Since both $x^{*}$ and $z^{*}$ are global minima of $f$, then $f\left(x^{*}\right)=f\left(z^{*}\right)$. By the definition of $f$, we also have $f\left(x^{*}\right) \leq F_{x^{*}}\left(U^{*}\right), f\left(z^{*}\right) \leq F_{z^{*}}\left(\widehat{U}^{*}\right)$. This implies that

$$
F_{x^{*}}\left(U^{*}\right)+F_{z^{*}}\left(\widehat{U}^{*}\right) \leq \frac{1}{2}\left(f\left(x^{*}\right)+f\left(z^{*}\right)\right) \leq \frac{1}{2}\left(F_{x^{*}}\left(U^{*}\right)+F_{z^{*}}\left(\widehat{U}^{*}\right)\right),
$$

a contradiction.

\section{References}

[1] S. Alama and M. Del Pino, 'Solution of elliptic equations with indefinite nonlinearities via Morse theory and linking', Ann. Inst. H. Poincaré, Anal. Non Linéaire 13 (1996), 95-115.

[2] A. Ambrosetti, V. Felli and A. Malchiodi, 'Ground states of nonlinear Schrödinger equations with potentials vanishing at infinity’, J. Eur. Math. Soc. 7 (2005), 117-144.

[3] H. Brezis and E. Lieb, 'A relation between pointwise convergence of functions and convergence of functions', Proc. Amer. Math. Soc. 88 (1983), 486-490.

[4] D. G. Costa and H. Tehrani, 'Existence of positive solutions for a class of indefinite elliptic problems in $\mathbb{R}^{N}$, Calc. Var. Partial Differential Equations 13 (2001), 159-189. 
[5] B. Gidas, W.-M. Ni and L. Nirenberg, 'Symmetry of positive solutions of nonlinear elliptic equations in $\mathbb{R}^{N}$, Adv. Math. Suppl. Stud. 7A (1981), 369-402. (Math. Anal. Appl. Part A).

[6] W.-M. Ni and I. Takagi, 'On the shape of least-energy solutions to a semilinear Neumann problem', Comm. Pure Appl. Math. XLIV (1991), 819-851.

[7] P. H. Rabinowitz, 'On a class of nonlinear Schrödinger equations', Z. Angew. Math. Phys. 43 (1992), 270-291.

[8] , Minmax Methods in Critical Point Theory with Applications to Differential Equations, CBMS Regional Conference Series in Mathematics, 65 (American Mathematical Society, Providence, RI, 1986).

[9] X.-F. Wang, 'On concentration of positive bound states of nonlinear Schrödinger equations', Comm. Math. Phys. 153 (1993), 229-244.

[10] X.-F. Wang and B. Zeng, 'On concentration of positive bound states of nonlinear Schrödinger equations with competing potential functions', SIAM J. Math. Anal. 28 (1997), 633-655.

JING LONG, Wuhan Institute of Physics and Mathematics,

Chinese Academy of Sciences, P.O. Box 71010, Wuhan 430071

and

Graduate School, Chinese Academy of Sciences, Beijing 100049, People's Republic of China

e-mail: alongjing@yahoo.com.cn

JIANFU YANG, Department of Mathematics, Jiangxi Normal University, Nanchang, Jiangxi, 330022, People's Republic of China

e-mail: jfyang_2000@yahoo.com 